\title{
Frequências de estimulação tátil em cordeiros lactentes: influência no ganho médio de peso, temperamento e relação homem-animal
}

\author{
Frequencies of tactile stimulation in suckling lambs: influence on \\ average weight gain, temperament and human-animal relationship
}

\author{
Luciane Erzinger $^{1}$, Tâmara Duarte Borges ${ }^{2 *}$, Saulo Henrique Weber ${ }^{2}$, Philippe Winagraski ${ }^{1}$, Nathaniele Penso \\ Gonçalves Viana ${ }^{1}$, Melissa Vink ${ }^{1}$, Cristina Santos Sotomaior ${ }^{2}$ \\ ${ }^{1}$ Medicina Veterinária, Pontifícia Universidade Católica do Paraná (PUCPR), Curitiba, PR, Brasil \\ 2 Programa de Pós-graduação em Ciência Animal (PPGCA), Pontifícia Universidade Católica do Paraná (PUCPR), Curitiba, PR, Brasil
}

\section{Resumo}

A estimulação tátil precoce em animais tem como benefícios a redução do estresse, melhoras no manejo, nos índices zootécnicos e no relacionamento ser-humano animal; no entanto, há escassos estudos relacionados ao benefício da estimulação tátil em cordeiros. 0 presente estudo teve como objetivo testar diferentes frequências de estimulação tátil em cordeiros lactentes, avaliando o temperamento, ganho de peso e relação ser humano- animal. Trinta e seis cordeiros, do segundo dia de vida até o desmame (60 a 63 dias de vida), foram distribuídos em três tratamentos: tratamento controle ( $\mathrm{TC} ; \mathrm{n}=12$ ) - cordeiros que não receberam estímulo tátil; T3x $(\mathrm{n}=12)$ - estimulação tátil nos cordeiros três vezes por semana; e T5x (n = 12) estimulação tátil nos cordeiros cinco vezes por semana. Semanalmente os cordeiros eram pesados e o ganho de peso médio diário (GPD) calculado. Para a avaliação do temperamento durante a pesagem, eram avaliados a reatividade (REA, possibilidade de 5-escores) e o número de vocalizações na balança (VOC). Após a pesagem, era avaliada a velocidade de saída (VS). Ao desmame, os cordeiros foram submetidos a testes de arena: teste de isolamento social (TIS), teste de distância de fuga (TDF) e teste de aproximação forçada (TAF). Não houve diferença $(p>0,05)$ entre os tratamentos para as variáveis de temperamento mensuradas (REA, VOC, VS) e para o GPD. Não foram verificadas diferenças $(p>0,05)$ entre os tratamentos para o número de vocalizações durante o TIS. Os animais do T5x, porém, aproximaram-se mais do ser humano $(\mathrm{p}<0,05)$ no TDF e se deixaram tocar em menor tempo ( $\mathrm{p}<0,05)$ durante o TAF, em comparação ao TC. Concluiuse que variações na frequência de estimulação tátil (3 ou $5 \mathrm{x} /$ semana) não demostraram diferença no GPD e no temperamento de cordeiros estimulados do nascimento ao desmame. Entretanto, a estimulação feita cinco vezes por semana demonstrou melhores resultados durante os testes de relação ser humano-animal, com diminuição da distância de fuga e maior aproximação com o homem.

Palavras-chave: Ovinos. Bem-estar animal. Desempenho animal. 


\section{Abstract}

Animal early tactile stimulation has the benefits of reduced stress, improved handling, zootechnical indexes, and better human-animal relationship. There are few studies related to tactile stimulation benefits in lambs. Therefore, this experiment aimed to test different frequencies of tactile stimulation in suckling lambs, evaluating the temperament, weight gain and human-animal relationship. Thirty-six lambs from the second day of life until weaning (60 to 63 days of life) were divided into three treatments: control (TC; $n=12)$ - lambs that received no tactile stimulation; $T 3 x$ $(n=12)$ - lambs received tactile stimulation three times per week; and T5x $(n=12)$-lambs received tactilestimulation five times per week. Lambs were weighed weekly and the average daily weight gain ( $A D G$ ) was calculated. For the evaluation of temperament during the weighing, the reactivity (REA, 5-scores-possibilities) and the number of vocalizations in the scale (VOC) were evaluated. After weighing, the flight speed (FS) was evaluated. At weaning, the lambs were submitted to three arena-tests: social isolation test (SIT); flight-distance test (FDT) and forced approach test (FAT). There were no differences $(p>0.05)$ between the treatments for the temperament variables measured: REA, FS, VOC, and neither for $A D G$. No differences ( $p>0.05)$ were found between treatments for the number of vocalizations during SIT. However, T5x lambs were closer to humans $(p<0.05)$ in FDT and allowed to be touched sooner $(p<0.05)$ during FAT than did TC lambs. It was concluded that variations in the frequency of tactile stimulation ( 3 or $5 x /$ week) showed no difference in ADG and temperament of lambs stimulated from birth to weaning. However, the stimulation done five times a week demonstrated better results during the human-animal relationship tests, with a decrease in the flight distance and greater approximation with man.

Keywords: Sheep. Animal welfare. Animal performance.

\section{Introdução}

A ovinocultura está presente em praticamente todos os continentes. Sua expansão no mundo foi significante, principalmente por se adaptar a diferentes climas, relevos e vegetações (Viana, 2008). No Brasil, a produção ovina envolve atualmente a quantidade de 18.410 .551 animais.
Só no estado do Paraná, 598.264 ovinos são criados com a finalidade principal de produção de carne (IBGE, 2016).

Independente dos tipos de sistemas empregados (extensivo, semi-intensivo e intensivo), é muito importante para a produção ovina a implementação de boas práticas de manejo, visando o bem-estar do rebanho (Goddard, 2006). Estudos que buscaram uma melhoria no bem-estar dos animais, com foco na diminuição da reatividade animal e manutenção de uma boa relação ser humano-animal, têm demonstrado também melhorias na qualidade da carne (Lensink et al., 2000), diminuição da mortalidade de cordeiros (Napolitano et al., 2005,2008 ) e aumento da fertilidade de machos (Hemsworth et al., 1986).

A estimulação tátil na infância basicamente tem efeito no sistema nervoso central (SNC), uma vez que o desenvolvimento do SNC dos animais é um processo dinâmico que depende de interações do meio antes e após o nascimento (Lupien et al., 2009). Dependendo da espécie animal, existem diferentes tipos de desenvolvimento cerebral ao nascer, divididos em altriciais e precociais (Fox, 1966). As espécies altriciais, como os cães, gatos e roedores, nascem com um maior grau de imaturidade cerebral, sendo totalmente dependentes da mãe. Os precociais, como os bovinos, ovinos e equinos, são mais maduros e têm melhor desenvolvimento do SNC, pois já nascem com a capacidade de andar (Fox, 1966).

Em herbívoros, a primeira socialização e estabelecimento do vínculo do filhote com a mãe acontece logo após o nascimento, quando a mãe realiza o comportamento de limpar e lamber o filhote, servindo para a estimulação e secagem do cordeiro (Barros et al., 2011). Com o passar do tempo, o filhote começa a interagir com outros cordeiros, iniciando a socialização secundária, e é neste momento que a interação ser humano-animal é muito importante para que haja a formação de vínculo com o homem (Oliveira, 2013).

Desta forma, a estimulação tátil tem sido usada como ferramenta para o estabelecimento de uma boa relação ser humano-animal, reduzindo significativamente o medo dos animais frente ao homem, tornando-os mais dóceis (Boivin et al., 2000). Pesquisas clássicas com estimulação tátil 
em ratos demostraram benefícios no ganho de peso (McClelland, 1956; Levine, 1956, 1957, 1960). Com outros mamíferos, como roedores (Kurosawa et al., 1995), bovinos (Schmied et al., 2008) e equinos (Lynch et al., 1974), os resultados positivos envolveram mudanças de comportamento: menor distância de fuga, diminuição do estresse e do comportamento de ansiedade. Para animais de produção, no entanto, principalmente ovinos e suínos, os benefícios da estimulação tátil não são facilmente detectados (Oliveira, 2013) e poucos estudos têm sido desenvolvidos.

Desta forma, o presente trabalho visou avaliar diferentes frequências de estimulação tátil sobre o ganho médio de peso diário e temperamento de cordeiros, além de verificar o estabelecimento de uma boa relação ser humano-animal.

\section{Material e métodos}

0 estudo foi realizado no setor de ovinocultura da Fazenda Experimental Gralha Azul, da Pontifícia Universidade Católica do Paraná (PUCPR), e foi aprovado por Comitê de Ética no Uso de Animais (CEUA) desta mesma instituição, sob número de protocolo 01071.

Foram acompanhados 31 partos, totalizando o nascimento de 36 cordeiros utilizados para o presente experimento. Os manejos realizados no primeiro dia de vida foram: a) garantia de que todos os cordeiros tivessem acesso ao colostro da mãe até as seis primeiras horas pós-nascimento; b) cura do umbigo com iodo a $10 \%$; c) identificação dos animais; d) pesagem ao nascer.

Os animais permaneceram junto as suas mães durante todo o experimento e tiveram acesso ao creep feeding desde os primeiros dias de idade, com fornecimento de concentrado comercial à vontade. Mães e filhotes permaneciam em piquetes de pasto nativo e, no final da tarde (17 h), eram recolhidos em um aprisco para passar a noite, onde recebiam silagem de milho e feno Tifton (Cynodon spp.), caracterizando sistema semi-intensivo.

Para o presente experimento, os 36 cordeiros foram acompanhados desde o segundo dia de vida até o desmame (entre 60 e 63 dias de vida) e foram divididos em três tratamentos:
- TC: tratamento controle ( $\mathrm{n}=12 ; 7$ machos e 5 fêmeas), composto por cordeiros que não receberam nenhum tipo de estímulo e seguiram o manejo de rotina da fazenda;

- T3x: tratamento estimulação tátil dos cordeiros três vezes por semana (segunda, quarta e sextafeira) ( $\mathrm{n}=12 ; 7$ machos e 5 fêmeas).

- T5x: tratamento estimulação tátil dos cordeiros cinco vezes por semana (segunda, terça, quarta, quinta e sexta-feira) ( $\mathrm{n}=12 ; 7$ machos e 5 fêmeas).

Para os procedimentos de estimulação tátil dos tratamentos T3x e T5x, mães e cordeiros eram conduzidos para o aprisco. Logo em seguida, os cordeiros eram separados das mães e permaneciam em baia adjacente (mesma baia destinada ao creep feeding). 0 estímulo tátil era realizado por estudantes de veterinária, previamente treinados, que se sentavam no chão e posicionavam o cordeiro entre as pernas, limitando o espaço do animal com o braço e mão. Com a palma da mão aberta, realizavam movimentos contínuos de estimulação desde a cabeça do animal até a cauda, respeitando a frequência de uma estimulação por segundo, pelo período total de 3 minutos para cada cordeiro.

Semanalmente, todos os 36 cordeiros eram pesados e submetidos à avaliação de temperamento. Entende-se por temperamento o conjunto de reações dos animais frente a situações novas e ameaçadoras, principalmente as relacionadas ao homem, que desencadeiam comportamento de medo (Maffei, 2009). Utilizando tal abordagem, foi possível realizar a avaliação desta característica, qualificando-se a reação do animal diretamente com os seguintes testes: escore de reatividade (REA) e velocidade de saída da balança (VS).

0 escore de REA (composto pelos escores de movimentação e tensão) e o número de vocalizações eram iniciados logo após o fechamento do portão da balança, e a observação do comportamento do cordeiro era feita pelo período total de 10 segundos, conforme descrito na Tabela 1. As vocalizações também eram contadas dentro de tal intervalo de tempo.

Após a pesagem dos animais, o teste de VS era aplicado. Para a realização deste teste, marcou-se o tempo (cronômetro Digital Incoterm T-TIM-0010.00) que o cordeiro levou para percorrer uma distância específica (1 metro) logo após a saída 
do portão da balança; maiores valores indicam pior temperamento, pois demonstram que os animais saíram mais rápido da balança, remetendo ao medo em relação aos seres humanos.

Tabela 1 - Descrição do escore de reatividade e número de vocalizações aplicados durante o procedimento de pesagem

\begin{tabular}{|c|c|}
\hline Variáveis & Descrição dos escores \\
\hline \multirow[t]{5}{*}{ MOV } & 1 - Nenhuma movimentação \\
\hline & $\begin{array}{l}2 \text { - Pouca movimentação, durante menos da metade do } \\
\text { período de observação }\end{array}$ \\
\hline & $\begin{array}{l}3 \text { - Movimentação frequente (durante metade ou mais } \\
\text { da metade do período de observação) }\end{array}$ \\
\hline & $\begin{array}{l}4 \text { - Movimentos constantes e vigorosos (durante todo } 0 \\
\text { período de observação) }\end{array}$ \\
\hline & $\begin{array}{l}5 \text { - Movimentos constantes e vigorosos; animal pode } \\
\text { saltar, levantar as patas dianteiras ou ajoelhar }\end{array}$ \\
\hline \multirow[t]{4}{*}{ TEN } & $\begin{array}{l}1 \text { - Animal não realiza movimentos rápidos de cabeça } \\
\text { e pescoço }\end{array}$ \\
\hline & $\begin{array}{l}2 \text { - Animal exibe pouca movimentação de cabeça e } \\
\text { pescoço }\end{array}$ \\
\hline & $\begin{array}{l}3 \text {-Animal exibe movimentos constantes e vigorosos de } \\
\text { cabeça e pescoço }\end{array}$ \\
\hline & $\begin{array}{l}4 \text { - Animal aparenta estar "paralisado", podendo ter } \\
\text { tremores musculares }\end{array}$ \\
\hline REA & Soma dos escores de MOV e TEN \\
\hline VOC & Contagem do número de vocalizações \\
\hline
\end{tabular}

Nota: REA = somatória dos escores de movimentação (MOV) e tensão (TEN); VOC = número de vocalizações na balança.

Com o peso dos cordeiros obtidos semanalmente, era calculado o ganho de peso diário (GPD), considerando-se a diferença de peso vivo entre as duas avaliações, dividida pelo intervalo de dias entre estas.

Após o desmame dos animais (entre 60 e 63 dias de vida), todos os cordeiros dos três grupos foram submetidos aos testes de arena, com o intuito de avaliar a relação ser humano-animal. Utilizou-se, para tal, uma arena de $2 \times 2,70$ metros, ao lado da baia do creep feeding. Os testes de arena utilizados foram:

a) Teste de isolamento social (TIS): o cordeiro era colocado sozinho na baia (arena) durante o período total de um minuto, e o número de vocalizações era mensurado neste intervalo de tempo.

b) Teste de distância de fuga (TDF): também realizado dentro da baia (arena), de forma individual, o TDF mede a distância que um observador pode se aproximar do animal até que este reaja. Sendo assim, um avaliador previamente treinado andava em direção ao cordeiro, de forma perpendicular ao animal, com passadas regulares (aproximadamente 1 passo por segundo) e os braços junto ao corpo. No momento em que o animal reagia à aproximação (com afastamento ou deslocamento), a posição do animal era fixada e a distância da pessoa era medida com o auxílio de uma trena. Quando o animal se deixava ser tocado, a distância de fuga era considerada como zero.

c) Teste de aproximação forçada (TAF): com o objetivo de mensurar o medo dos animais aos seres humanos, o avaliador entrava na baia (arena) e se posicionava dentro da arena ao lado da porta pelo período inicial de 5 segundos. Logo em seguida, após o animal perceber a sua presença, iniciava-se o movimento em direção ao cordeiro com o intuito de tocá-lo na região da paleta. Media-se a latência para o primeiro toque e, em casos em que os cordeiros não se deixavam tocar, o tempo total mensurado era de 2 minutos (tempo total do teste).

\section{Análise estatística}

As comparações entre o número de vocalizações na balança (VOC), velocidade de saída (VS) e ganho de peso diário (GPD), entre os três tratamentos, foram realizadas pela análise de variância (ANOVA), seguida do teste de Tukey, com nível de significância de 5\%. Para os testes de arena (TIS, TDF e TAF) e de REA foi realizado o teste de Kruskal-Wallis, seguido pelo teste de MannWhitney, com nível de significância de 5\%. Para todas as análises foi utilizado o software estatístico Statgraphics versão 4.1 .

\section{Resultados}

Não houve diferença $(p>0,05)$ entre os tratamentos para as variáveis de temperamento mensuradas (REA, VS, VOC) e nem para o GPD (Tabela 2). 
Tabela 2 - Mediana ( \pm desvio padrão) dos escores de reatividade e média ( \pm desvio padrão) da velocidade de saída, vocalização na balança e ganho de peso médio diário para os tratamentos TC, T3x e T5x

\begin{tabular}{ccccc}
\hline Tratamento & REA & VS (m/s) & VOC ( $n)$ & GPD (kg/dia) \\
\hline TC & $5 \pm 1,67$ & $0,89 \pm 1,01$ & $1,80 \pm 1,12$ & $0,254 \pm 0,12$ \\
T3x & $5 \pm 1,43$ & $0,90 \pm 0,75$ & $2,06 \pm 1,34$ & $0,256 \pm 0,12$ \\
T5x & $5 \pm 1,29$ & $0,95 \pm 0,78$ & $2,02 \pm 1,06$ & $0,253 \pm 0,13$ \\
\hline
\end{tabular}

Nota: $\mathrm{TC}=$ tratamento controle. $\mathrm{T} 3 \mathrm{x}=$ estimulação tátil 3 vezes por semana; $T 5 x$ = estimulação tátil 5 vezes por semana. REA = escore de reatividade; VS = velocidade de saída; $V O C=$ número de vocalizações na balança; $G P D=$ ganho de peso médio diário. Para VS, VOC e GPD, utilizou-se ANOVA seguido de Tukey. Para REA realizou-se Kruskal-Wallis, seguido pelo teste de MannWhitney (nível de significância $p<0,05$ ).

As mensurações da velocidade de saída e vocalização ao longo do tempo demonstram que os cordeiros, nas semanas iniciais, foram mais rápidos para percorrer o caminho pós-balança e também vocalizaram mais (Figuras 1 e 2), independente do tratamento $(\mathrm{p}<0,05)$.

As mensurações do GPD ao longo do tempo indicaram que o TC foi o que obteve as maiores amplitudes de perdas e ganhos de peso (Figura 3).

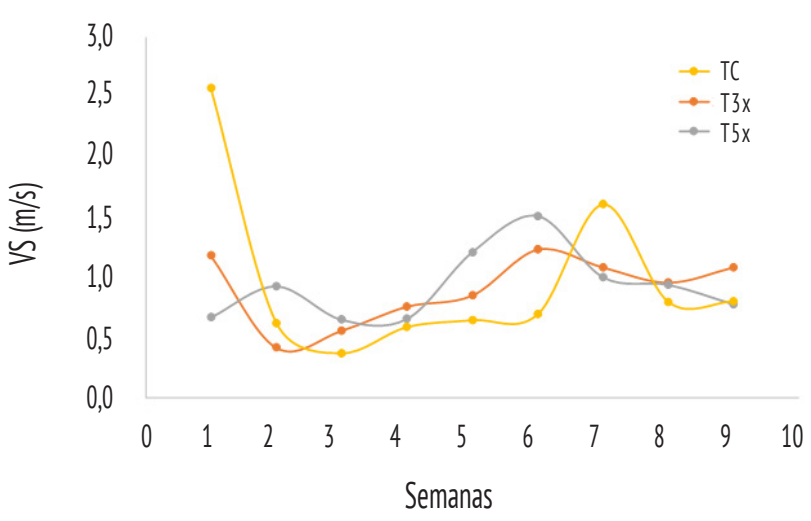

Figura 1 - Velocidade de saída ao longo do tempo (semanas de vida) para os tratamentos TC, T3x e T5x.

Nota: $V S$ = velocidade de saída. $T C$ = tratamento controle. $T 3 x$ = estimulação tátil três vezes por semana; $T 5 x$ = estimulação tátil cinco vezes por semana.

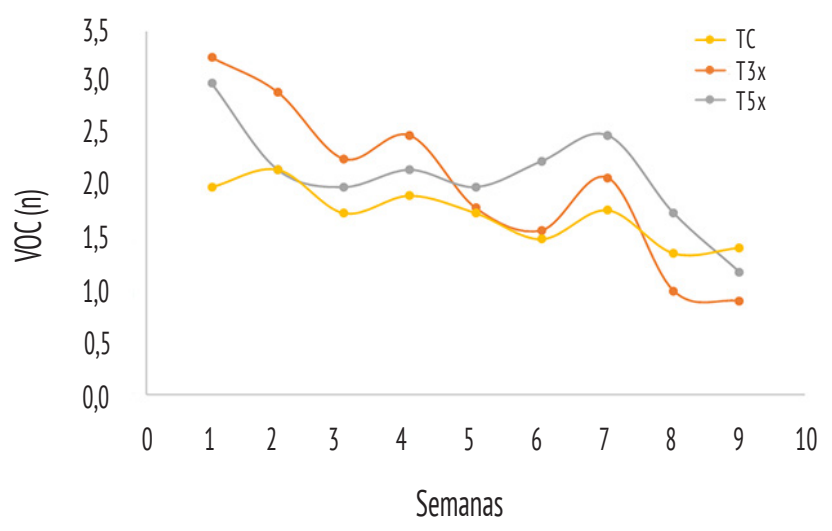

Figura 2 - Número de vocalizações (n) ao longo do tempo (semanas de vida) para os tratamentos TC, T3x e T5x.

Nota: VOC = número de vocalizações. $\mathrm{TC}=$ tratamento controle. $\mathrm{T} 3 \mathrm{x}=$ estimulação tátil três vezes por semana; $T 5 x$ = estimulação tátil cinco vezes por semana.

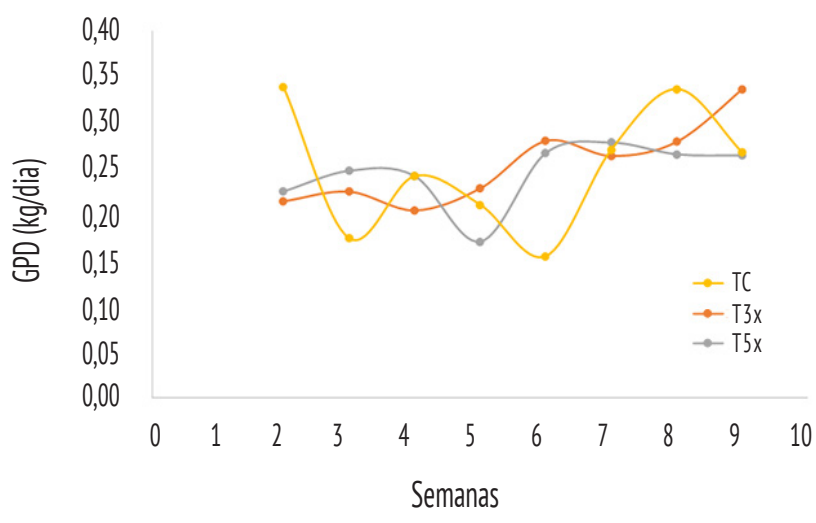

Figura 3 - Ganho de peso diário (Kg/dia) ao longo do tempo (semanas de vida) para os tratamentos TC, T3x e T5x.

Nota: $\mathrm{GPD}=$ ganho de peso diário. $\mathrm{TC}=$ tratamento controle. $\mathrm{T} 3 \mathrm{x}=$ estimulação tátil três vezes por semana; $T 5 x=$ estimulação tátil cinco vezes por semana.

Não foram encontradas diferenças $(\mathrm{p}>0,05)$ entre os tratamentos para o número de vocalizações durante o TIS. No entanto, em comparação ao TC, os animais do T5x se aproximaram mais do ser humano $(p<0,05)$ no TDF e se deixaram tocar em menor tempo $(\mathrm{p}<0,05)$ durante o TAF, como demonstrado na Tabela 3. 
Tabela 3 - Número médio ( \pm erro padrão) de vocalizações durante 0 teste de isolamento social, distância máxima de aproximação durante 0 teste de distância de fuga, e latência para 0 primeiro toque durante 0 teste de aproximação forçada para os tratamentos TC, T3x e T5x

\begin{tabular}{cccc}
\hline & TIS & TDF & TAF \\
\cline { 2 - 4 } Tratamento & VOC ( $)$ & D (cm) & TT (seg) \\
TC & $16,65 \pm 1,13^{\mathrm{a}}$ & $85,25 \pm 15,30^{\mathrm{a}}$ & $63,40 \pm 12,30^{\mathrm{a}}$ \\
T3x & $16,61 \pm 1,63^{\mathrm{a}}$ & $58,92 \pm 18,18^{\mathrm{ab}}$ & $25,60 \pm 9,76^{\mathrm{ab}}$ \\
T5x & $14,84 \pm 1,11^{\mathrm{a}}$ & $33,57 \pm 12,49^{\mathrm{b}}$ & $21,80 \pm 9,26^{\mathrm{b}}$ \\
\hline
\end{tabular}

Nota: $\mathrm{TC}=$ tratamento controle. $\mathrm{T} 3 \mathrm{x}=$ estimulação tátil 3 vezes por semana; $\mathrm{T} 5 \mathrm{x}=$ estimulação tátil 5 vezes por semana. TIS = teste de isolamento social; VOC = vocalizações; TDF = Teste de distância de fuga; $D$ = distância; TAF = teste de aproximação forçada; TT= latência para o primeiro toque. Letras diferentes na coluna indicam diferença estatística entre os tratamentos pelo teste de Kruskal-Wallis seguido por Mann-Whitney $(p<0,05)$.

\section{Discussão}

A relação ser humano-animal está ligada a vários aspectos de bem-estar (Probst et al., 2012), uma vez que os animais introduzem o ser humano na sua rede social (Rault et al., 2011). A reatividade de um animal pode aumentar ou diminuir dependendo dos manejos adequados ou inadequados a que é submetido, e muitos dos problemas têm relação com o medo (Vandenheede et al., 1993).

Para melhorar a relação positiva entre ser humano e animal, o contato tátil neonatal tem auxiliado no sentido de aumentar a sociabilidade, deixando os animais mais calmos (Freitas, 2014), além de apresentar resultados secundários como o incremento no ganho de peso médio (Hemsworth et al., 1986; Lensink et al., 2000; Verwer et al., 2009). Especificadamente para cordeiros, poucos estudos visando entender os efeitos da estimulação tátil têm sido desenvolvidos (Barbosa et al., 2001; Gómez, 2007), e nenhum estudo com foco nas frequências de dias de estimulação foi realizado até então.

No presente estudo, o GPD dos animais e as variáveis de temperamento mensuradas durante os procedimentos de pesagem (número de vocalizações, escore de reatividade e velocidade de saída) foram iguais entre os tratamentos. Em contrapartida, Napolitano et al. (2005), em estudo pioneiro aplicando estimulação tátil em cordeiros nos 14 primeiros dias de vida, encontraram resultados satisfatórios para o grupo estimulado, sendo que este obteve maiores ganhos de peso, além de promover um declínio mais lento do $\mathrm{pH}$ da carne post-mortem. Já Oliveira (2013), trabalhando com cordeiros da raça Santa Inês, encontrou ganho de peso até $14 \%$ maior em animais estimulados por meio de escovação. Caroprese et al. (2006) também reportaram que animais estimulados retornaram mais rapidamente aos níveis basais de cortisol após manuseio e isolamento social, além de manterem melhor função imunológica.

Uma possível explicação para os resultados encontrados neste experimento poderia estar relacionada ao manejo particular da Fazenda Gralha Azul, da PUCPR, que já tem como premissa manter bom manejo alimentar, sanitário e de relação ser humano-animal. No entanto, verifica-se na Figura 3 que o tratamento controle foi o que obteve maiores amplitudes de variação de peso, o que pode também caracterizar uma maior dificuldade na adaptação quando comparado com os animais estimulados (T3x e T5x), que mantiveram uma estabilidade de GPD ao longo do tempo.

Duranteostestesparaavaliação do temperamento dos animais, observou-se que os cordeiros de uma forma geral vocalizavam mais e saíam mais rápido da balança nas primeiras semanas (Figuras 1 e 2). Comportamentos estes que foram decaindo ao longo do tempo, o que remete ao fato de os animais se acostumarem com o procedimento de pesagem e manejo rotineiro da fazenda.

Para os testes de arena, não foi encontrada diferença entre os tratamentos quando aplicado o teste de isolamento social e contado o número de vocalizações. Por tratar-se de animais gregários (Lynch et al., 1992), os ovinos demonstram muito medo quando isolados, vocalizando mais (Le Neindre et al., 1996). Além disso, o fato de o presente estudo utilizar cordeiros ainda recém-desmamados durante o teste pode ter contribuído para o aumento das vocalizações (resposta comportamental indicativa de estresse), o que, de acordo com Walser et al. (1982), tem relação com a comunicação entre mãe e filhote, que é muito utilizada por cordeiros para chamar a atenção da mãe e, assim, forçar um encontro. 
Para o teste de distância de fuga e para o teste de aproximação forçada foram encontrados resultados significativos, sendo que animais estimulados cinco vezes por semana obtiveram as menores distâncias em relação ao ser humano e permitiram ser tocados em menor tempo. Tal dado demonstra que o vínculo entre humano e animal foi melhor estabelecido quando se tinham maiores chances de contato positivo (T5x). Além disso, tal relação mais íntima com o animal durante a fase de amamentação demonstrou reduzir o medo em relação ao ser humano. Resultados semelhantes foram obtidos por Napolitano et al. (2005), em que cordeiros tiveram menor medo em relação ao ser humano perante o "teste humano estacionário".

Uma relação humana-animal positiva traz diversos benefícios dentro de um sistema de produção animal, facilitando manejos de rotina como palpação, condução, procedimentos de coleta de amostras e exames clínicos gerais (Boivin et al., 2000). Observa-se, também, uma tendência de melhora nos níveis imunológicos dos animais, com redução de doenças (Lensink et al., 2000), o que também está relacionado a uma melhor adaptação do cordeiro ao período de desmame (Caroprese et al., 2006). Além disso, caracteriza um sistema com boas práticas de manejo e aplicação de premissas de bem-estar animal que, no Brasil, vem ganhando cada vez mais espaço pelo interesse dos produtores em ampliar mercados mais exigentes e também pela sociedade, que vem buscando maiores informações sobre os sistemas de criação ao qual os animais são submetidos (Molento et al., 2005).

\section{Conclusão}

Conclui-se que variações na frequência de estimulação tátil (três ou cinco vezes por semana) não demostraram diferença no ganho de peso e temperamento de cordeiros estimulados do nascimento ao desmame. No entanto, a estimulação feita cinco vezes na semana demonstrou melhores resultados durante os testes de relação ser humanoanimal, com diminuição da distância de fuga e maior contato com o homem.

\section{Agradecimentos}

Os autores agradecem aos alunos do curso de Medicina Veterinária e do Programa de Pós-Graduação em Ciência Animal (PPGCA), da Pontifícia Universidade Católica do Paraná (PUCPR), que auxiliaram na estimulação tátil dos cordeiros do presente estudo.

\section{Referências}

Araújo Filho JA. Aspectos zooecológico e agropecuários do caprino e do ovino nas regiões semi-áridas. Sobral, CE: Embrapa Caprinos e Ovinos; 2006.

Barbosa OR, Macedo FAF, Groes RV, Guedes JMF. Zoneamento bioclimático da ovinocultura no estado do Paraná. Rev Bras Zootec. 2001; 30(2):454-60.

Barros CS, Monteiro ALG, Dittrich JR, Fernandes MAM, Pinto S. Comportamento social de ovinos: apresentação de técnicas. Sci Agrar Paran. 2011;10(2):5-20.

Boivin X, Tournadre H, Le Neindre P. Hand-feeding and gentling influence early-weaned lambs'attachment responses to their stockperson. J Anim Sci. 2000;78(4): 879-84.

Caroprese M, Napolitano F, Albenzio M, Annicchiarico G, Musto M, Sevi A. Influence of gentling on lamb imune response and human-lamb interactions. Appl Anim Behav Sci. 2006;99(1-2):118-31.

Ensminger ME, Parker RO. Sheep and goat science. Danville: Interstate Printers \& Publishers; 1986. 643 p.

Fox MW. Neuro-behavioral ontogeny. A synthesis of ethological and neurophysiological concepts. Brain Res. 1966;2(1):3-20.

Freitas ACB. Avaliação do temperamento de ovinos com treinamento de estímulo tátil e deslocamento com auxílio de cabresto [dissertação]. Nova Odessa: Instituto de Zootecnia; 2014. 73 p.

Goddard P, Waterhouse T, Dwyer C, Stoot A. The perception of the welfare of sheep in extensive systems. Small Rumin Res. 2006;62(3):215-25. 
Gómez JMD. Temperamento de três raças de ovinos submetidos a diferentes manejos de pastagem [dissertação]. Porto Alegre: Universidade Federal do Rio Grande do Sul; 2007. 113 p.

Hemsworth PH, Barnett JL, Hansen C. The influence of handling by humans on the behaviour, reproduction and corticosteroids of male and female pigs. Appl Anim Behav Sci. 1986;15(4):303-14.

IBGE. Instituto Brasileiro de Geografia e Estatística. Pesquisa da Pecuária Municipal. [acesso 12 ago 2018]. Disponível em: https://tinyurl.com/y8uk8o4l.

Kurosawa M, Lundeberg T, Agren G, Lund I, Uvnäs-Moberg K. Massage-like stroking of the abdomen lowers blood pressure in anesthetized rats: influence of oxytocin. J Auton Nerv Syst. 1995;56(1-2):26-30.

Le Neindre P, Boivin X, Boissy A. Handling of extensively kept animals. Appl Anim Behav Sci. 1996;49(1):73-81.

Lensink BJ, Boivin X, Pradel P, Le Neindre P, Veissier I. Reducing veal calves' reactivity to people by providing additional human contact. J Anim Sci. 2000;78(5):1213-8.

Levine S. Further study of infantile handling and adult avoidance learning. J Pers. 1956;25(1):70-80.

Levine S. Infantile experience and resistance to physiological stress. Science. 1957;126(3270):405.

Levine S. Stimulation in infancy. Sci Am. 1960;202:81-6.

Lupien SJ, McEwen BS, Gunnar MR, Heim C. Effects of stress throughout the lifespan on the brain, behaviour and cognition. Nat Rev Neurosci. 2009;10(6):434-45.

Lynch JJ, Fregin GF, Mackie JB, Monroe Jr RR. Heart rate changes in the horse to human contact. Psychophysiology. 1974;11(4):472-8.

Lynch JJ, Hinch GN, Adams DB. The behaviour of sheep: biological principles and implications for production. Wallingford: CAB International; 1992. 237 p.

Maffei WE. Reatividade animal. R Bras Zootec. 2009; 38(spe):81-92.
McClelland WJ. Differential handling and weight gain in the albino rat. Can J Exp Psychol.1956;10(1):19-22.

Molento CFM. Bem-estar e produção animal: aspectos econômicos - Revisão. Arch Vet Sci. 2005;10(1):1-11.

Napolitano F, De Rosa G, Sevi A. Welfare implications of artificial rearing and early weaning in sheep. Appl Anim Behav Sci. 2008;110(1-2):58-72.

Napolitano F, Marino R, Musto M, Caternolo G, Sevi A. Effects of gentling on behavior and meat quality of lambs. Ital J Anim Sci. 2005; 4(Suppl 2):357-9.

Oliveira D. Potenciais tátil no comportamento e desenvolvimento de cordeiros e leitões [tese]. Jaboticabal: Universidade Estadual Paulista; 2013. 93 p.

Probst JK, Neff AS, Leiber F, Kreuzer M, Hillmann E. Gentle touching in early life reduces avoidance distance and slaughter stress in beef cattle. Appl. Anim Behav. Sci. 2012;139(1-2):42-9.

Rault JL, Boissy A, Boivin X. Separation distress in artificially-reared lambs depends on human presence and the number of conspecifics. Appl Anim Behav Sci. 2011;132(1-2):42-50.

Schmied C, Waiblinger S, Scharl T, Leisch F, Boivin X. Stroking of different body regions by a human: Effects on behavior and heart rate of dairy cows. Appl Anim Behav Sci. 2008;109(1):25-38.

Vandenheede M, Bouissou MF. Sex diferences in fear reactions in sheep. Appl Anim Behav Sci. 1993;37(1):39-55.

Verwer CM, Van Amerongen G, van den Bos R, Hendriksen CFM. Handling effects on body weight and behaviour of group-housed male rabbits in a laboratory setting. Appl Anim Behav Sci. 2009;117(1-2):93-102.

Viana JGA. Panorama geral da ovinocultura no mundo e no Brasil. 2008 [acesso 12 ago 2018]. Disponível em: https://tinyurl.com/ybxymwjc.

Walser ES, Walters E, Hague P. Vocal communication between ewes and their own and alien lambs. Behaviour. 1982;81(2):140-51. 